\title{
ENCAIXE PESSOA-ORGANIZAÇÃO: UM ESTUDO EM UMA MULTINACIONAL DO SETOR MÉDICO-HOSPITALAR
}

\section{FIT PERSON ORGANIZATION: A STUDY IN A MULTINATIONAL IN THE MEDICAL-HOSPITAL SECTOR}

\author{
Murilo Alberto Franco \\ Fundação Getúlio Vargas - FGV - EAESP \\ murilo.franco@abbott.com
}

Anderson de Souza Sant'Anna Fundação Getúlio Vargas - FGV - EAESP anderson.santanna@fgv.br

Daniela Martins Diniz

Fundação Dom Cabral - FDC - MG danidiniz09@yahoo.com.br

Aurea de Fátima Oliveira Universidade Federal de Uberlândia - UFU - MG aurea.oliveira@ufu.br

Submissão: $29 / 09 / 2000$

Aprovação: 15/06/2021

\section{RESUMO}

Adotando a perspectiva interacionista do encaixe pessoa-organização, este artigo apresenta resultados de estudo destinado a verificar a congruência entre valores pessoais e organizacionais percebidos por profissionais inseridos em uma multinacional da área médicohospitalar. Quanto ao método, foi desenvolvida uma pesquisa survey envolvendo a aplicação de 241 questionários junto à profissionais de diferentes níveis hierárquicos da organização. Os resultados indicam que a análise da congruência entre valores pessoais e organizacionais, calculada por meio da medida de encaixe P-O, apresentou-se positiva para todos os grupos avaliados (gerencial, técnico-administrativo e operacional), indicando um encaixe acima do nível médio. Quanto aos valores pessoais, os achados apontam "benevolência" e "conformidade" como os tipos mais recorrentes, em contraposição a "estimulação" e "poder". Quanto aos valores organizacionais registra-se consenso entre os profissionais de que a organização prioriza aqueles relacionados às categorias de "domínio" e de "prestígio organizacional". Quanto à contribuição, este estudo corrobora a viabilidade de pesquisas envolvendo o construto encaixe P-O no Brasil, considerando-se a escassez de estudos nacionais na área.

Palavras-chave: Valores Organizacionais. Valores Pessoais. Encaixe Pessoa-Organização. 


\section{ABSTRACT}

Adopting the interactionist perspective of the person-organization fit, this article presents results of a study aimed at verifying the congruence between personal and organizational values of professionals inserted in a multinational in the medical-hospital area. As for the method, a survey research was carried out involving the application of 241 questionnaires with professionals from different hierarchical levels of the organization. The results indicate that the analysis of the congruence between personal and organizational values, calculated using the P-O fit measure, was positive for all the groups evaluated (managerial, technicaladministrative and operational), indicating a fit above the average level. As for personal values, the findings point to "benevolence" and "conformity" as the most recurrent types, as opposed to "stimulation" and "power". As for organizational values, there is consensus among professionals that the organization prioritizes those related to the categories of "domain" and "organizational prestige". As for the contribution, this study corroborates the feasibility of research involving the $\mathrm{P}-\mathrm{O}$ fitting construct in Brazil, considering the scarcity of national studies in the area.

Keywords: Organizational Values. Personal Values. Personal-Organization Fitting.

\section{INTRODUÇÃO}

Por que indivíduos em uma mesma organização demonstram níveis diferentes de engajamento no e com o trabalho? Por que pessoas inseridas em um mesmo setor ou equipe, muitas vezes sob a mesma supervisão apresentam graus distintos de comprometimento? Diante dessas indagações, a questão da congruência entre valores pessoais e organizacionais ganha relevância no contexto das organizações contemporâneas, em face da sua estreita relação com a produtividade individual e organizacional, como apontam diversos estudos na área (PASCHOAL; TAMAYO, 2003; SANT'ANNA; MORAES; KILIMNIK, 2003; 2005; MEDEIROS; OLIVEIRA, 2007).

$\mathrm{O}$ aumento da preocupação em torno desse tema decorre também das constantes transformações por quais a relação indivíduo-trabalho-organização tem passado na última década. Isso se observa notadamente a partir das mudanças recentes decorrentes da transição para a quarta revolução industrial (SCHWAB, 2016), que passa a se configurar como uma variável contextual que afeta a relação entre os interesses da organização e os interesses dos seus membros (SANT'ANNA; FERREIRA; SANTOS, 2020).

Suportada por avanços sem precedentes das tecnociências e das neurociências, a quarta revolução industrial gera novos padrões de competitividade expressos em modelos de negócios virtuais, em plataformas e redes, assim como em modelos de gestão cada vez mais flexíveis, assentados em múltiplos vínculos de trabalho cada vez mais descentralizados, senão precarizados. Implica, igualmente, na demanda por ressignificação de categorias analíticas clássicas - trabalho, profissão, carreira, valores-, que passam a ser fortemente afetadas pelas novas tecnologias de base digital, integradas por sensores, robôs e algoritmos autorreferenciados (SCHWAB, 2016; ROSS, 2017; SUSSKIND, SUSSKIND, 2017).

No Brasil, pesquisas sobre a relação entre o indivíduo e a organização têm sido realizadas sob diferentes enfoques, como os estudos sobre o significado do trabalho (ANDRADE; MOURÃO, 2001), sentidos do trabalho (OLIVEIRA et al., 2004; PRADO et al., 2020), valores pessoais (PASCHOAL; TAMAYO, 2003), valores organizacionais (BORGES, 2005; ALVES; CARVALHO, 2020), congruência entre valores pessoais e 
organizacionais (TEPECI; BARTLETT, 2002; DELFINO et al., 2010; SANTOS, 2016; RODRIGUEZ et al., 2020), entre outros.

De acordo com Tepeci (2001) e Tepeci e Barlett (2002), pesquisas no campo das organizações tradicionalmente buscam explicar o comportamento e a motivação dos funcionários com base nas características da organização (situacionistas) ou do indivíduo (personalistas). A primeira perspectiva, a situacional, baseia-se na premissa de que o ambiente organizacional é a principal variável que explica o comportamento dos indivíduos no ambiente de trabalho. Já a perspectiva personalista sugere que as características individuais como personalidade, valores, crenças, entre outras, são as principais influenciadoras do comportamento dos indivíduos nas organizações (TEPECI; BARLETT, 2002).

$\mathrm{O}$ debate entre essas perspectivas tem, mais recentemente, suscitado o desenvolvimento de estudos que visam articulá-las. Nessa linha, o engajamento dos indivíduos com a organização passa a ser compreendido como a complexa interação entre características pessoais e aspectos do ambiente organizacional (TEPECI, 2001; 2011; DELFINO et al., 2010; RODRIGUEZ et al., 2020). Assim, a perspectiva interacionista ganha espaço por meio de suas contribuições nos estudos sobre Encaixe Pessoa-Organização ou simplesmente encaixe P-O (P-O fit). Tais pesquisas se fundamentam na noção de que a congruência entre características dos indivíduos e das organizações é desejável para ambas as partes: indivíduo e organização (ROBERT; WASTI, 2002; TEPECI; BARTLETT, 2002).

No âmbito desses estudos, o encaixe P-O tem sido investigado a partir da análise da congruência entre valores pessoais (VP) e valores organizacionais (VO). Isso, pois, os "valores" representam um dos principais componentes da cultura organizacional que guiam o comportamento dos membros da organização (KRISTOFF, 1996; TEPECI; BARTLETT, 2002; TEPECI, 2011). Além disso, valores desempenham um importante papel tanto no alcance dos objetivos organizacionais quanto no atendimento das necessidades do indivíduo (OLIVEIRA; TAMAYO, 2004; OLIVEIRA; 2004).

Entretanto, VP e VO podem ser conflitantes, tensão que pode afetar o engajamento do indivíduo no trabalho, bem como o seu desempenho no exercício da sua função, afetando, consequentemente, o desempenho da organização (SILVA, 2004; TAMAYO, 2005). Desse modo, o estudo da congruência entre VO e VP pode contribuir para um melhor entendimento da dinâmica de harmonia e conflito entre as organizações e seus membros e o efeito disso sobre o comportamento do indivíduo, além das consequências para o desempenho da organização.

Numa consulta ao Scientific Periodicals Electronic Library (SPELL) da Associação Nacional de Pós-Graduação e Pesquisa em Administração (ANPAD), considerando como palavras-chave "P-O Fit" e expressões similares (como encaixe P-O, alinhamento P-O, congruência VP e VO), foi identificada somente uma publicação relacionada ao tema proposto neste artigo (RODRIGUEZ et al., 2020), o que é um indicador da relevância deste estudo. Além disso, estudo bibliométrico de Santos (2016) demonstrou como são escassos os estudos sobre o construto "congruência entre indivíduo e organização" no contexto brasileiro.

Com base na abordagem interacionista (Encaixe P-O) é delineado o objetivo da pesquisa, a qual visa investigar a congruência entre valores pessoais e organizacionais percebidos por profissionais inseridos em uma multinacional da área médico-hospitalar. Para tanto, foi necessário: a) identificar e mensurar o perfil de valores pessoais dos trabalhadores dos três grupos funcionais pesquisados (nível gerencial, técnico-administrativo e operacional); b) Identificar e mensurar os tipos de valores organizacionais percebidos pelos três grupos funcionais pesquisados; c) calcular a medida de encaixe P-O para os três grupos funcionais pesquisados, a partir da congruência entre VP e VO. 


\section{REFERENCIAL TEÓRICO}

\subsection{Valores Pessoais (VP) e Organizacionais (VO)}

De modo geral, a literatura considera os valores pessoais como aspectos fundamentais que orientam as escolhas dos indivíduos e os valores organizacionais como guias do funcionamento da organização. Logo, os valores representam variáveis-chave à análise dos comportamentos individual e organizacional. Mais especificamente, valores pessoais podem ser definidos como crenças duradouras e internalizadas no indivíduo sobre maneiras desejáveis ou indesejáveis de se comportar, tendo relação com a noção de obrigação moral (MEGLINO; RAVLIN, 1998). Já os valores organizacionais são princípios que guiam a vida organizacional e representam aquilo que é considerado "certo" e "errado" em uma organização, orientando os comportamentos dos indivíduos no ambiente de trabalho (TAMAYO, 2001; SCHEIN, 2009).

$\mathrm{Na}$ literatura sobre o tema é possível encontrar alguns instrumentos para a mensuração de valores pessoais e organizacionais (MEGLINO, RAVLIN, 1998; PASCHOAL; TAMAYO, 2003; PORTO, 2005; MARTINS; SANTOS, 2006; ALVES; CARVALHO, 2020). Todavia, parte considerável dos estudos adota um único instrumento para medir os dois tipos de valores, o que na visão de Oliveira e Tamayo (2004) é uma limitação de pesquisa por serem construtos distintos. Desse modo, neste estudo foram adotados dois instrumentos diferentes para mensurar os VP e os VO.

Para tal, foi adotado o Inventário de Valores de Schwartz (SVS) para a mensuração de valores pessoais. Trata-se de um instrumento validado a partir de uma pesquisa empírica transcultural aplicada em mais de 60 países, demonstrando o seu potencial de universalidade (PASCHOAL; TAMAYO, 2003; SCHWARTZ, 2005; DELFINO et al., 2010). No Brasil, particularmente, o Inventário de Valores de Schwartz (1992) foi validado por Tamayo e Schwartz (1993). Para Rohan (2000), o modelo de valores de Schwartz (1992) é uma contribuição crucial para o entendimento dos componentes do sistema de valores humanos.

Para Schwartz (1992), valores pessoais são representações cognitivas de três exigências humanas universais: necessidades biológicas do indivíduo, necessidades sociais de interação e necessidades socio-constitucionais de sobrevivência e bem-estar dos grupos. Para que possa lidar com a realidade, o indivíduo deve reconhecer tais necessidades e buscar respostas adequadas para satisfazê-las, o que se dá por meio de formas específicas, estabelecidas social e culturalmente (SCHWARTZ, 1992, 2005; OLIVEIRA; TAMAYO, 2004).

Com base nessas exigências humanas universais, Schwartz (1992) desenvolve um modelo teórico contendo dez tipos de valores reunidos no Quadro 1.

Quadro 1 - Tipos motivacionais de valores

\begin{tabular}{|c|l|l|}
\hline $\mathbf{N}^{\circ}$ & \multicolumn{1}{|c|}{ Tipos } & \multicolumn{1}{c|}{ Exemplos de Valores } \\
\hline 1 & Autodeterminação & Criatividade, Liberdade, Independência \\
\hline 2 & Estimulação & Excitação, Ousadia \\
\hline 3 & Hedonismo & Prazer \\
\hline 4 & Realização & Ambição, Competência \\
\hline 5 & Poder & Autoridade, Poder Social \\
\hline 6 & Segurança & Ordem social \\
\hline 7 & Conformidade & Obediência, Polidez, Respeito \\
\hline 8 & Tradição & Respeito, Moderação \\
\hline 9 & Benevolência & Preocupação com o coletivo, Honestidade \\
\hline 10 & Universalismo & Justiça Social, Igualdade \\
\hline & & Fonte: Adaptado de Schwartz (1992, 2005).
\end{tabular}

Nesse estudo, Schwartz (1992) confirmou, ainda, as hipóteses de que cinco valores 
servem a interesses individuais (poder, realização, hedonismo, estimulação e autodeterminação) e três tipos de valores servem a interesses coletivos (benevolência, tradição e conformidade). Já os valores de universalismo e segurança apresentam-se como interesses mistos, localizados nos interstícios entre interesses individuais e coletivos. $\mathrm{O}$ autor indica ainda relações entre compatibilidades e conflitos decorrentes da articulação simultânea de diferentes grupos de valores. Desse modo, são compatíveis os grupos de poder e realização, de realização e hedonismo, de hedonismo e estimulação, de estimulação e autodeterminação, de autodeterminação e universalismo, de universalismo e benevolência, de tradição e conformidade, de conformidade e segurança; de segurança e poder. Já conflitos são esperados quando esses grupos de valores são priorizados: autodeterminação e estimulação, estimulação e conformidade, tradição e segurança; universalismo e benevolência, realização e poder; hedonismo e conformidade; e, conformidade e tradição (SCHWARTZ, 1992; ALVES; CARVALHO, 2020).

As oposições entre tipos de valores podem entendidas também quando os tipos motivacionais são organizados em duas dimensões bipolares: "abertura a mudanças versus conservação" e "autopromoção versus autotranscendência". A "abertura a mudanças" versus "conservação" contrapõe valores que enfatizam pensamento e ação independente (estimulação, autodeterminação e hedonismo), favorecendo a mudança, a valores que dão ênfase à auto-restrição, preservação de práticas tradicionais e proteção da estabilidade (segurança, conformidade e tradição). A segunda dimensão contrasta "autopromoção" versus "autotranscendência", capturando o conflito existente entre a busca do próprio sucesso e do domínio sobre os outros (poder e realização), com valores que enfatizam a aceitação dos outros como iguais e a preocupação com seu bem-estar (universalismo e benevolência). Hedonismo tem tanto elementos de abertura à mudança quanto de autopromoção (SCHWARTZ, 1992; ALVES; CARVALHO, 2020).

Ressalta-se, ainda, que a busca simultânea dos seguintes tipos motivacionais pode gerar conflitos sociais e psicológicos: a) autodeterminação e estimulação versus segurança, tradição e conformidade - como os primeiros tipos motivacionais enfatizam pensamentos e ações independentes, podem ocorrer conflitos com auto-restrição, preservação de práticas tradicionais e proteção da estabilidade; b) universalismo e benevolência versus realização e poder - a preocupação com o bem-estar dos outros e sua aceitação como iguais contrapõe e interfere na busca de sucesso e domínio sobre os outros; c) hedonismos versus conformidade e tradição - satisfazer os próprios desejos conflita com a aceitação dos limites impostos externamente (SCHWARTZ, 1992; ALVES; CARVALHO, 2020).

Esses tipos motivacionais, as relações de conflito e compatibilidade, também foram confirmados em estudo posterior de Schwartz (2005), realizado em 67 países envolvendo 64.271 participantes. O autor acrescenta que os indivíduos diferem na importância que atribuem a cada um dos dez tipos motivacionais. Ou seja, a hierarquia de valores dos indivíduos é diferente e expressa as suas concepções daquilo que é bom para ele próprio, para a sociedade e para a organização na qual ele trabalha.

O Inventário de Perfis de Valores Organizacionais (IPVO) é um modelo teórico clássico na área utilizado para mensurar os valores organizacionais (OLIVEIRA; TAMAYO, 2004; DELFINO et al., 2010; ALVES; CARVALHO, 2020). Foi desenvolvido e validado por Oliveira e Tamayo (2004), autores que se basearam no modelo de Schwartz (1992).

O IPVO é composto por oito tipos de valores que sinalizam o perfil cultural de uma organização (ver, Quadro 2). 
Quadro 2 - Tipos de valores do IPVO

\begin{tabular}{|l|l|}
\hline \multicolumn{1}{|c|}{ Valores } & \multicolumn{1}{c|}{ Descrição } \\
\hline 1) Autonomia & Indica a abertura que a organização oferece para a participação dos empregados \\
\hline $\begin{array}{l}\text { 2) Bem-estar do } \\
\text { empregado }\end{array}$ & $\begin{array}{l}\text { Indica a preocupação da organização em propiciar satisfação ao empregado, atentando-se } \\
\text { para a qualidade de vida no trabalho. }\end{array}$ \\
\hline 3) Realização & $\begin{array}{l}\text { Indica a busca da organização pelo alcance das metas, bem como a aplicação das } \\
\text { competências organizacionais e individuais }\end{array}$ \\
\hline 4) Domínio & $\begin{array}{l}\text { Inclui itens relativos ao poder, cuja meta central é a obtenção de status e de controle sobre } \\
\text { pessoas e recursos, bem como a busca de uma posição dominante no mercado. }\end{array}$ \\
\hline 5) Prestígio & Indica a busca da organização pelo alcance de legitimidade e respeito da sociedade \\
\hline 6) Tradição & $\begin{array}{l}\text { Indica a busca da organização pela preservação e respeito aos costumes e práticas } \\
\text { legitimadas historicamente }\end{array}$ \\
\hline 7) Conformidade & $\begin{array}{l}\text { Inclui orientações e limites para o comportamento dos empregados no ambiente de } \\
\text { trabalho }\end{array}$ \\
\hline 8) Ética & Indica a preocupação da organização com questões sociais, coletivas e externas à empresa \\
\hline
\end{tabular}

No desenvolvimento do IPVO, Oliveira e Tamayo (2004) testaram, empiricamente, uma hipótese de aderência entre os dois sistemas de valores (pessoais e organizacionais). Com base nos resultados do estudo, concluíram que a simetria motivacional dos VP e VO tem suporte teórico e empírico, o que implica contribuição significativa à noção de "Encaixe $\mathrm{P}$ O”.

\subsection{Encaixe Pessoa-Organização (Encaixe P-O)}

A pesquisa sobre "Encaixe P-O", em essência, direciona-se à compreensão dos antecedentes e consequentes da compatibilidade entre pessoas e organizações. Para Kristof (1996), o encaixe P-O consiste na congruência entre congruência entre pessoas e organizações que ocorre quando uma entidade provém o que a outra necessita e elas compartilham características e valores similares.

Analisar o encaixe P-O a partir da investigação da congruência entre VP e VO é uma das formas muito adotadas pelos estudos na área, dado que valores são características fundamentais e duradouras tanto de indivíduos como de organizações (MEGLINO; RAVLIN, 1998; TEPECI; BARTLETT, 2002; ROBERT; WASTI, 2002).

$\mathrm{O}$ encaixe $\mathrm{P}-\mathrm{O}$, via congruência de valores, pode ser medido de forma direta ou indireta. Na medição direta os indivíduos são questionados quanto a similaridades entre seus valores e os da organização (TEPECI; BARTLETT, 2002). Kristof (1996), todavia, adverte que características de valores não descritas de forma explícita e objetiva podem não assegurar que dimensões compatíveis sejam consideradas.

Diante disso, a medição indireta, adotada na maioria dos estudos sobre o tema, utiliza dimensões compatíveis entre si, nas quais valores individuais e organizacionais são avaliados sob as mesmas categorias, permitindo análises comparativas mais confiáveis. Em outros termos, os indivíduos são, inicialmente, solicitados a descrever sua percepção quanto à cultura organizacional (TEPECI; BARTLETT, 2002). Na sequência, a partir das mesmas categorias, os indivíduos descrevem a cultura "desejada", ou seja, o que as pessoas esperam de um ambiente de trabalho. O encaixe P-O, então, pode ser avaliado como a soma das diferenças entre os perfis de pontuação ou por meio da correlação entre os perfis de pontuação, conhecida como correlação de Spearman ( $Q$ score), aspectos aprofundados na próxima seção.

\section{MÉTODO}

Tomando como referência o objetivo do estudo, foi desenvolvida pesquisa de natureza quantitativa por meio da técnica de survey, (GONÇALVES; MEIRELLES, 2004), 
possibilitando a medição objetiva dos VP e VO e o cálculo da medida de encaixe P-O (CASTRO, 2005).

A unidade de análise da pesquisa foi composta por profissionais de nível gerencial, técnico-administrativo e operacional de multinacional da área médico-hospitalar. Trata-se de uma empresa fundada há mais de 32 anos que produz dispositivos tecnológicos médicohospitalares comercializados no Brasil e exportados para ampla gama de países. Pelo fato de ser uma organização intensiva em tecnologia e fortemente ligada aos campos da biotecnologia/ nanotecnologia, considerou-se ser um caso relevante para a realização da pesquisa. Além disso, é uma organização que investe pesado em pesquisa, ciência, tecnologia e inovação, modelo típico de organizações do padrão de competitividade exigido no contexto da quarta-revolução industrial.

Dois outros critérios também foram considerados para a escolha da organização investigada: porte da empresa que possibilitou um universo adequado para pesquisa; facilidade de acesso à organização, seus profissionais e informações.

O universo de pesquisa compreendeu 241 profissionais vinculados a três grupos funcionais: Gerencial (ocupantes de cargo de chefia, totalizando 21 pessoas entre diretores, coordenadores e gerentes); Técnico-administrativo (40 profissionais que executam atividades relacionadas aos processos produtivos e tarefas administrativas: contábil, recursos humanos, tecnologia da informação); Operacional (formada por 180 respondentes de nível operacional).

Quanto aos instrumentos de coleta de dados, foram adotados dois modelos de questionário: o Inventário de Valores de Schwartz (1992) para a mensuração dos valores pessoais (com escala Likert de 7 pontos) e o IPVO de Oliveira e Tamayo (2004) para a mensuração dos valores organizacionais (com escala Likert de 6 pontos). Além disso, foram coletados dados profissionais e pessoais dos respondentes. Ressalta-se que foi aplicado um pré-teste dos instrumentos em um grupo de seis funcionários com o objetivo de verificar o grau de entendimento das questões e da semântica utilizada.

Para uniformizar o mesmo número de categorias nos dois instrumentos adotados, foi necessário realizar um tratamento preliminar dos dados. Com isso, a categoria de "segurança" presente somente no modelo de VP foi desconsiderada. Ademais, Oliveira e Tamayo (2004) realizaram os seguintes grupamentos: "autodeterminação" e "estimulação" correspondentes a "autonomia"; "benevolência" e "universalismo" correspondentes a "coletividade"; e "domínio" e "prestígio" correspondentes a "poder".

Quanto à técnica de tratamento dos dados, foi feita inicialmente uma análise exploratória dos mesmos envolvendo um esforço de identificação e tratamento de problemas inerentes aos dados (TABACHNICK; FIDEL, 2001). Uma primeira etapa envolveu a descrição das estimativas básicas das variáveis para a verificação de inconsistências na entrada de dados. Assim, buscando dirimir dúvidas quanto aos valores univariados dos dados, calculou-se a média e o desvio-padrão das variáveis ao nível agregado da amostra no pacote estatístico SPSS 13.0. Posteriormente, foi analisado o percentual de dados ausentes, ou seja, questões sem respostas (HAIR JR. et al., 1998), que se mostrou bastante baixo (93 ocorrências que representaram $0,52 \%$ dos dados coletados).

Com o intuito de verificar a normalidade univariada, empregou-se a avaliação gráfica com testes formais de assimetria e normalidade. Pela análise gráfica foi possível a verificação de padrões de normalidade expressos nos gráficos dos quantis observados e esperados da distribuição normal, conhecido como gráfico Q-Q (PESTANA; GAGEIRO, 2003). Prosseguindo à avaliação de problemas inerentes aos dados, foi feita a identificação de observações extremas (outliers), casos com valores muito discrepantes dos restantes dos respondentes (KLINE, 1998). Para tanto, foi feita uma classificação dos valores extremos que estavam fora do limite de três vezes o intervalo interquartil para o primeiro e terceiro quartil, tendo sido encontradas 57 respostas extremas. Aplicando a sugestão de Kline (1998) e 
Tabachnick e Fidel (2001), tais respostas foram substituídas por outras válidas para as escalas, mas com valores menos ofensivos em relação às normas do banco de dados.

Posteriormente, foi feito o cálculo do encaixe $\mathrm{P}-\mathrm{O}$ a partir da análise da congruência entre VP e VO utilizando três procedimentos: i) a soma das diferenças - $|\mathrm{D}|$; ii) a soma do quadrado das diferenças - $\mathrm{D}^{2}$; iii) o cálculo do Q-score de Spearman (KRISTOF, 1996; MEGLINO; RAVLIN, 1998). A soma das diferenças - |D $\mid$, considera a soma das distâncias absolutas obtida pela soma dos desvios absolutos entre os valores organizacionais e os valores pessoais (ver primeira fórmula na tabela abaixo). O segundo procedimento de cálculo considera a soma dos desvios quadráticos, conforme segunda fórmula apresentada na Tabela 1.

Tabela 1 - Procedimentos de cálculo

\begin{tabular}{c|c} 
Primeiro procedimento de cálculo & Segundo procedimento de cálculo \\
$\sum_{i=1}^{k}\left|V O_{k}-V P_{k}\right|=\sum_{i=1}^{k}\left|D_{k}\right|$ & $\sum_{i=1}^{k}\left(V O_{k}-V P_{k}\right)^{2}=\sum_{i=1}^{k}\left(D_{k}\right)^{2}$
\end{tabular}

Fonte: Elaborado pelos autores.

Quanto ao terceiro procedimento, foi calculada a medida de Spearman ( $Q$-score) entre os dois perfis de valores pessoais e organizacionais. A escolha pelo $Q$-score se baseou nos estudos de Kristof (1996) e de Tepeci e Bartlett (2002), que argumentam que é o método mais utilizado nas pesquisas de encaixe $\mathrm{P}-\mathrm{O}$ operacionalizadas via congruência de valores. Os dados obtidos a partir das técnicas apresentadas foram apresentados na próxima seção.

\section{RESULTADOS DE PESQUISA}

\subsection{Valores Pessoais e Organizacionais}

A pesquisa empírica contemplou trabalhadores de três grupos funcionais, sendo 21 ocupantes de cargos gerenciais (9\%), 40 de nível técnico-administrativo (17\%) e 180 de nível operacional (74\%). Quanto ao gênero, os resultados apontam que a maioria dos participantes é do sexo feminino (86\%) e quase todas as mulheres encontram-se no nível operacional (99\%).

Quanto à faixa etária, verificou-se predominância de jovens, tendo cerca de $70 \%$ com até 30 anos de idade, 24\% com idade de 31 a 40 anos e $6 \%$ com mais de 41 anos. Quanto ao nível de instrução, a maioria dos respondentes possui $2^{\circ}$ grau completo $(71 \%), 16 \%$ com curso superior completo, $8 \%$ com curso superior em andamento e $5 \%$ com $1^{\circ}$ grau completo. Houve predominância de indivíduos com formação superior completa no grupo gerencial (95\%). Já no grupo de profissionais de nível operacional registrou-se predominância de profissionais com o segundo grau completo (78\%). Sobre o tempo de atuação na empresa, a maioria dos respondentes $(60 \%)$ possui até 3 anos de casa.

A Tabela 5 contempla análise descritiva dos valores pessoais dos dez tipos motivacionais de Schwartz (1992).

Tabela 2 - Valores pessoais dos 10 tipos motivacionais

\begin{tabular}{l|c|c|c|c|c}
\hline \multirow{2}{*}{ Tipos Motivacionais } & \multicolumn{5}{|c}{ Total (N=241) } \\
\cline { 2 - 6 } & Mínimo & Máximo & Mediana & $\begin{array}{c}\text { Média } \\
\text { aritmética }\end{array}$ & $\begin{array}{c}\text { Desvio } \\
\text { Padrão }\end{array}$ \\
\hline Poder & 0,50 & 5,83 & 3,17 & 3,16 & 0,91 \\
\hline Realização & 0,50 & 6,83 & 4,00 & 3,96 & 0,91 \\
\hline Hedonismo & 1,33 & 6,67 & 4,33 & 4,21 & 1,06 \\
\hline Estimulação & $-0,67$ & 6,67 & 3,00 & 2,96 & 1,26 \\
\hline Autodeterminação & 2,86 & 6,00 & 4,71 & 4,60 & 0,73 \\
\hline
\end{tabular}




\begin{tabular}{l|c|c|c|c|c}
\hline Universalismo & 2,20 & 6,60 & 4,80 & 4,74 & 0,72 \\
\hline Benevolência & 2,70 & 6,50 & 5,20 & 5,17 & 0,64 \\
\hline Tradição & 1,40 & 6,60 & 4,00 & 4,05 & 0,86 \\
\hline Conformidade & 3,00 & 6,75 & 5,00 & 4,98 & 0,83 \\
\hline Segurança & 2,43 & 6,71 & 4,86 & 4,77 & 0,70 \\
\hline
\end{tabular}

Fonte: Dados da pesquisa.

Analisando os dados, constatou-se a predominância dos seguintes tipos para o universo pesquisado: benevolência $(5,17)$, conformidade $(4,98)$, segurança $(4,77)$, universalismo $(4,74)$, autodeterminação $(4,60)$, hedonismo $(4,21)$ e tradição $(4,05)$, todos com média aritmética acima de 4.

Uma análise comparativa dos tipos motivacionais entre os grupos revela a existência de diferenças estatisticamente significativas $(\mathrm{p}<0,05)$ para todos os tipos motivacionais investigados, com exceção de autodeterminação, conforme achados constantes na Tabela 3.

Tabela 3 - Valores pessoais dos tipos motivacionais (por grupo e total)

\begin{tabular}{|c|c|c|c|c|}
\hline \multirow[b]{2}{*}{ Tipos motivacionais } & \multicolumn{3}{|c|}{ Grupo Funcional } & \multirow[b]{2}{*}{$\begin{array}{c}\text { Total } \\
(\mathrm{N}=\mathbf{2 4 0})\end{array}$} \\
\hline & $\begin{array}{c}\text { Gerencial } \\
(A) \\
(\mathbf{N}=\mathbf{2 1})\end{array}$ & $\begin{array}{c}\text { Técnico-Adm } \\
(B) \\
(\mathbf{N}=\mathbf{4 0})\end{array}$ & $\begin{array}{c}\text { Operacional } \\
\text { (C) } \\
(\mathbf{N}=\mathbf{1 8 0})\end{array}$ & \\
\hline Poder & 2,82 & 3,3 & 3,16 & 3,24 \\
\hline Realização & 4,08 & 4,3 & 3,86 & 3,96 \\
\hline Hedonismo & 3,87 & 4,49 & 4,18 & 4,29 \\
\hline Estimulação & 2,84 & 3,43 & 2,87 & 3,02 \\
\hline Autodeterminação & 4,54 & 4,78 & 4,57 & 4,63 \\
\hline Universalismo & 4,29 & 4,67 & 4,80 & 4,73 \\
\hline Benevolência & 4,72 & 5,17 & 5,22 & 5,12 \\
\hline Tradição & 3,39 & 3,93 & 4,16 & 4,05 \\
\hline Conformidade & 4,32 & 5,12 & 5,03 & 4,98 \\
\hline Segurança & 4,27 & 4,69 & 4,85 & 4,77 \\
\hline
\end{tabular}

Fonte: Dados da pesquisa.

Os profissionais técnicos-administrativos manifestaram, para os tipos motivacionais poder e hedonismo, escores superiores aos observados para os profissionais de nível gerencial, os quais, por sua vez, não apresentaram diferenças significativas em relação aos trabalhadores de nível operacional. Escores superiores para os profissionais do nível técnico-administrativo também ocorreram para os tipos motivacionais de realização e estimulação em relação aos trabalhadores de nível operacional. Porém, estes últimos não apresentaram diferenças significativas em relação aos profissionais de nível gerencial.

Quanto ao tipo universalismo, os trabalhadores de nível operacional $(4,80)$ apresentaram escore significativamente superior aos profissionais de nível gerencial $(4,29)$, porém, não se diferenciaram em relação ao escore obtido pelos técnicos-administrativos $(4,67)$. Cabe destacar que para os tipos motivacionais benevolência, conformidade e segurança, não se observaram diferenças estatisticamente significativas entre os profissionais de nível operacional e técnico-administrativo. Ambos, porém, apresentaram escores significativamente superiores aos dos profissionais de nível gerencial.

Por fim, quanto ao tipo tradição, o grupo operacional $(4,16)$ apresentou escore significativamente superior ao dos profissionais de nível técnico-administrativo $(3,93)$. Estes, por sua vez, obtiveram escore significativamente superior ao dos profissionais de nível gerencial $(3,39)$.

Numa análise final, tem-se, portanto, que os técnicos-administrativos apresentaram, para os tipos realização e estimulação, escores significativamente superiores aos dos profissionais do grupo operacional, os quais foram superiores aos gerentes em relação aos 
tipos universalismo, benevolência, tradição, conformidade e segurança. Por último, os profissionais do grupo técnico-administrativo apresentaram, para os tipos motivacionais poder, hedonismo, benevolência, tradição, conformidade e segurança, escores significativamente superiores aos dos profissionais do grupo gerencial.

As diferenças e semelhanças entre os grupos funcionais em relação a cada tipo motivacional de VP podem visualizadas na Figura 1.

Figura 1 - Escore dos VP por grupo funcional

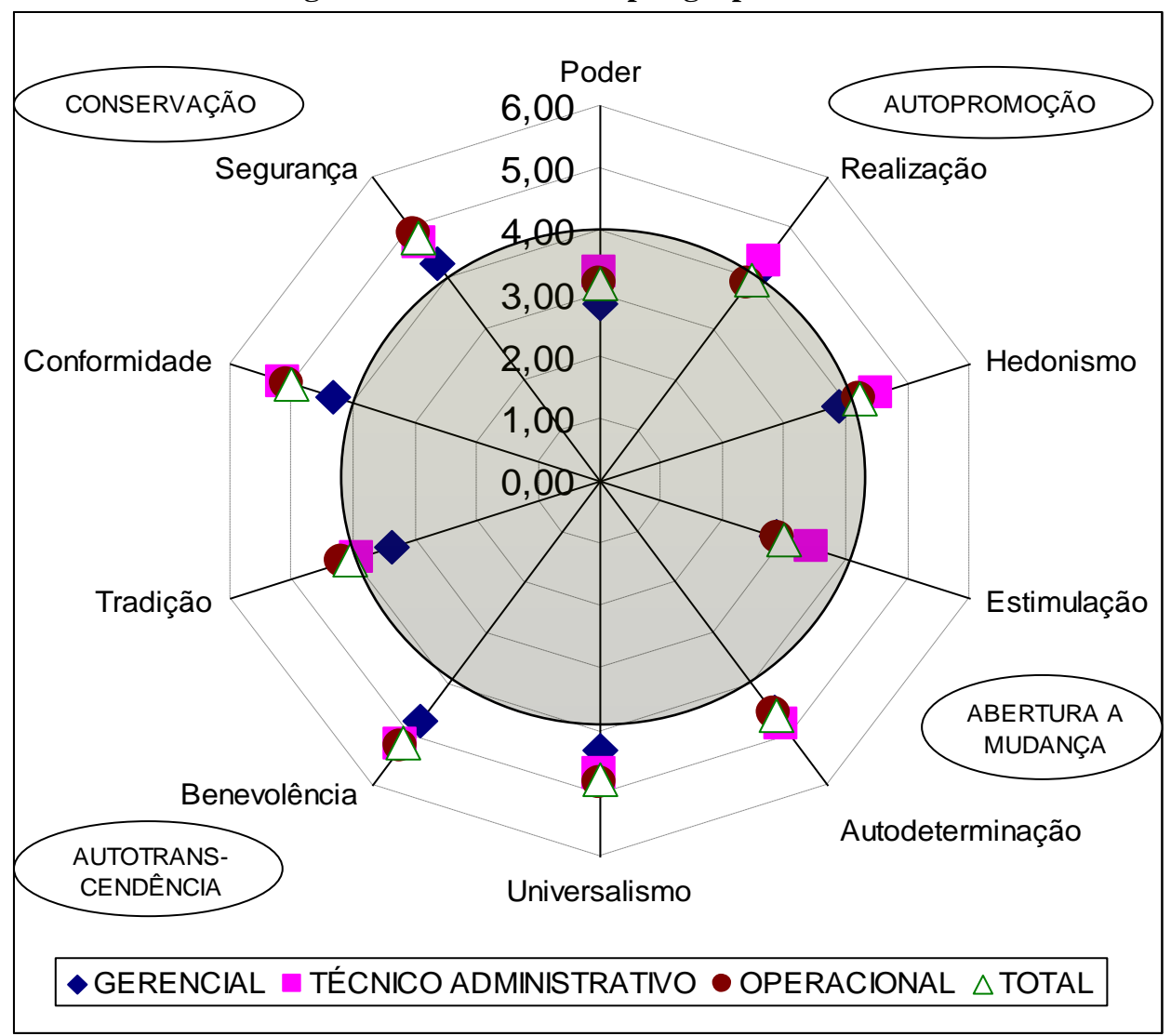

Fonte: Dados da pesquisa

Inicialmente, verificou-se que o nível gerencial se diferencia dos demais grupos funcionais ao apresentar escores inferiores para os tipos motivacionais contidos à esquerda do gráfico, os quais compõem, em sua maioria, os fatores de $2^{a}$ ordem (autotranscendência e conservação). Isso indica, portanto, que os profissionais de nível gerencial dão menos importância à aceitação dos outros como iguais e à preocupação com seu bem-estar (autotranscendência), bem como à preservação de práticas tradicionais e proteção da estabilidade (conservação).

Em relação aos tipos motivacionais contidos à direita do gráfico, os quais compõem os fatores de $2^{\mathrm{a}}$ ordem (autopromoção e abertura a mudanças), o grupo técnico-administrativo apresentou escores superiores na comparação com os demais grupos. Tal achado indica que os técnicos-administrativos dão mais importância a valores relacionados à busca pelo próprio sucesso e ao domínio sobre os outros.

Os dados apontam, ainda, que apesar das diferenças entre os escores em cada grupo funcional, a mesma tendência pode ser observada em relação aos grupos de valores. Ou seja, os três grupos funcionais tendem a ter escores mais altos e mais baixos em relação aos mesmos tipos motivacionais, manifestando, assim, o mesmo comportamento em relação 
àqueles tipos. Isto pode indicar que apesar das diferenças entre os grupos funcionais, inclusive quanto ao papel desempenhado na organização, no conjunto, os profissionais apresentam perfil de valores pessoais similares.

A análise descritiva dos VO que compõem o IPVO do modelo de Oliveira (2004) foi reunida na Tabela 4.

Tabela 4 - Categorias dos VO

\begin{tabular}{l|c|c|c|c|c}
\hline \multirow{2}{*}{ Categorias } & \multicolumn{5}{|c}{ Total (N=241) } \\
\cline { 2 - 6 } & Mínimo & Máximo & Mediana & $\begin{array}{c}\text { Média } \\
\text { aritmética }\end{array}$ & $\begin{array}{c}\text { Desvio } \\
\text { Padrão }\end{array}$ \\
\hline Realização & 2,00 & 6,00 & 4,80 & 4,68 & 0,83 \\
\hline Conformidade & 2,29 & 6,00 & 5,00 & 5,00 & 0,62 \\
\hline Domínio & 3,17 & 6,00 & 5,33 & 5,25 & 0,60 \\
\hline Bem-estar do empregado & 1,00 & 6,00 & 3,17 & 3,26 & 1,19 \\
\hline Tradição & 1,20 & 6,00 & 3,80 & 3,73 & 0,93 \\
\hline Prestígio Organizacional & 2,75 & 6,00 & 5,25 & 5,24 & 0,62 \\
\hline Autonomia & 1,25 & 5,88 & 3,94 & 3,82 & 0,97 \\
\hline Preocupação com a coletividade & 1,29 & 6,00 & 4,57 & 4,37 & 0,94 \\
\hline
\end{tabular}

Fonte: Dados da pesquisa.

Os achados apontam que os respondentes atribuem a seguinte ordem de importância em relação aos valores organizacionais: domínio $(5,25)$, prestígio organizacional $(5,24)$, conformidade $(5,00)$, realização $(4,68)$, preocupação com a coletividade $(4,37)$, autonomia $(3,82)$, tradição $(3,73)$ e bem-estar do empregado $(3,26)$. Nota-se, também, maior desviopadrão para esta última categoria quando comparado às demais, indicando menor consenso dos respondentes em relação à percepção de valores associados à preocupação da empresa em propiciar mais satisfação ao indivíduo.

Uma análise comparativa entre as categorias de VO revela diferenças significativas $(\mathrm{p}<0,05)$ entre os grupos funcionais pesquisados no que se refere às categorias realização, domínio, bem-estar do empregado, tradição, autonomia e preocupação com a coletividade. Quanto às demais categorias (conformidade e prestígio organizacional), não foram observadas diferenças estatisticamente significativas (Tabela 5).

Tabela 5 - Categorias dos VO: por grupo e total

\begin{tabular}{|c|c|c|c|c|}
\hline \multirow[b]{2}{*}{ Categorias } & \multicolumn{3}{|c|}{ Grupo Funcional } & \multirow{2}{*}{$\begin{array}{c}\text { Total } \\
(\mathrm{N}=240)\end{array}$} \\
\hline & $\begin{array}{c}\text { Gerencial } \\
(\mathrm{N}=\mathbf{2 1})\end{array}$ & $\begin{array}{l}\text { Técnico-Adm } \\
(\mathrm{N}=\mathbf{4 0})\end{array}$ & $\begin{array}{c}\text { Operacional } \\
(\mathrm{N}=\mathbf{1 8 0})\end{array}$ & \\
\hline Realização & 4,90 & $4,95^{\mathrm{c}}$ & 4,59 & 4,66 \\
\hline Conformidade & 4,99 & 5,06 & 4,99 & 5,01 \\
\hline Domínio & 4,90 & $5,25^{\mathrm{a}}$ & $5,29^{\mathrm{a}}$ & 5,24 \\
\hline Bem-estar do empregado & $3,97^{\mathrm{c}}$ & $3,72^{\mathrm{c}}$ & 3,08 & 3,28 \\
\hline Tradição & $3,98^{b}$ & 3,40 & $3,78^{b}$ & 3,68 \\
\hline Prestígio Organizacional & 5,20 & 5,22 & 5,25 & 5,20 \\
\hline Autonomia & $4,39^{\mathrm{c}}$ & $4,41^{\mathrm{c}}$ & 3,62 & 3,84 \\
\hline Preocupação com a coletividade & $5,13^{\mathrm{c}}$ & $4,83^{\mathrm{c}}$ & 4,18 & 4,39 \\
\hline
\end{tabular}

Fonte: Dados da pesquisa. OBS: Os valores marcados com letras indicam médias diferentes entre os pares considerados no nível de 5\% bicaudal (teste Mann-Whitney)

Para a categoria realização, os técnicos-administrativos apresentaram escore mais elevado $(4,95)$ que o observado junto aos profissionais de nível operacional $(4,59)$. Porém não foram observadas diferenças significativas daqueles em relação aos profissionais de nível gerencial $(4,90)$. Quanto ao domínio, os achados indicaram escores mais altos para os profissionais de nível operacional $(5,29)$ e administrativo $(5,25)$ em relação aos profissionais 
de nível gerencial $(4,99)$, muito embora os dois primeiros não apresentaram diferenças significativas entre si.

Já para bem-estar do empregado, autonomia e preocupação com a coletividade, verificou-se a inexistência de diferenças estatisticamente significativas entre os profissionais de nível gerencial e de nível técnico-administrativo. Por fim, em relação à categoria tradição, os resultados indicaram que tanto o grupo gerencial quanto o operacional apresentaram escores muito superiores aos técnicos-administrativos.

As diferenças entre os grupos em relação aos escores obtidos para cada uma das categorias do IPVO podem ser visualizadas na Figura 2.

Figura 2 - Categorias dos VO por grupo funcional

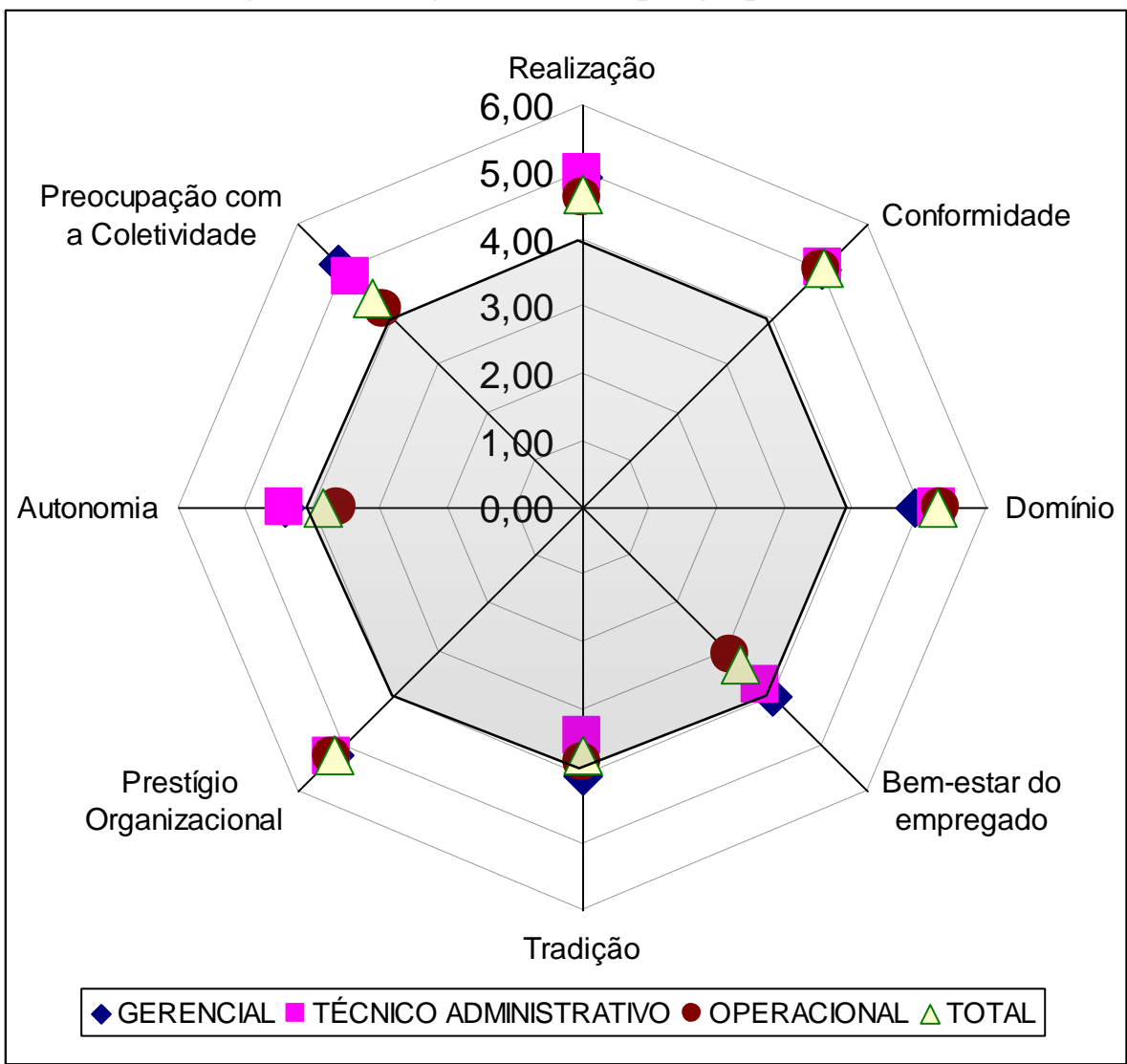

Fonte: Dados da pesquisa.

Com base na figura, nota-se, inicialmente, diferença significativa (a menor), entre os escores do grupo operacional quando comparados aos dos grupos gerencial e técnicoadministrativo em relação às categorias preocupação com a coletividade, autonomia e bemestar do empregado. Além disso, é visível o mais baixo escore, do grupo operacional, para esta última categoria, bem-estar do empregado, seguida de autonomia.

Em uma análise global, verifica-se homogeneidade dos profissionais dos três grupos pesquisados quanto às percepções de importância dos VO. Ou seja, apesar da diferença existente entre os escores de cada grupo funcional para cada uma das categorias, os mesmos apresentam de forma geral a mesma tendência de variação para a quase totalidade das categorias analisadas, ou seja, o mesmo comportamento de aumento e redução dos escores. Ademais, dois dos três mais altos escores, comuns aos grupos funcionais pesquisados, foram prestígio organizacional e conformidade. Tal achado indica uma percepção muito próxima dos profissionais dos três grupos de que a organização prioriza valores relacionados ao poder, 
prestígio, admiração e respeito da sociedade, bem como a definição de limites para as ações de seus membros.

De forma comum aos três grupos pesquisados, as categorias autonomia, tradição e bemestar do empregado aparecem com os mais baixos escores. Tal resultado indica que, na percepção dos profissionais, a organização dá menor prioridade a valores relacionados à busca de aperfeiçoamento do funcionário, à manutenção de costumes vigentes, bem como à qualidade de vida no trabalho.

\subsection{Encaixe Pessoa-Organização (P-O)}

Os resultados do cálculo da medida de encaixe P-O foram reunidos na Tabela 6.

Tabela 6 -Valores de encaixe P-O (Q-score - Spearman), por grupo e total

\begin{tabular}{l|c|c|c|c|c|c}
\hline Grupo & Amostra & Mínimo & Máximo & Mediana & $\begin{array}{c}\text { Média } \\
\text { Aritmética }\end{array}$ & $\begin{array}{c}\text { Desvio } \\
\text { Padrão }\end{array}$ \\
\hline Gerencial & 21 & $-0,29$ & 0,89 & 0,41 & 0,38 & 0,32 \\
\hline Técnico-Administrativo & 40 & $-0,64$ & 0,76 & 0,23 & 0,25 & 0,32 \\
\hline Operacional & 180 & $-0,82$ & 0,93 & 0,24 & 0,17 & 0,40 \\
\hline Total & 241 & $-0,82$ & 0,93 & 0,25 & 0,21 & 0,39 \\
\hline \multicolumn{7}{c}{ Fonte: dados da pesquisa. }
\end{tabular}

Analisando os dados, verificou-se que os escores do encaixe P-O apresentam-se positivos para todos os grupos funcionais investigados, indicando congruência entre os VP e VO acima do nível médio de encaixe (escore zero).

Em uma análise comparativa, verificou-se que o encaixe P-O para o nível gerencial foi o mais elevado $(0,38)$, revelando maior congruência entre seus valores pessoais e os valores da organização. Na sequência, em ordem decrescente, tem-se o grupo técnico-administrativo com encaixe P-O de 0,25. O mesmo, no entanto, não se verifica em relação ao grupo operacional $(0,17)$, cujo escore apresenta-se bastante inferior ao do grupo gerencial.

O valor da mediana obtida pelo grupo operacional $(0,24)$ bem acima da média $(0,17)$, indica que o valor do encaixe de pelo menos metade desses profissionais apresenta-se significativamente acima da média global. Tal achado, possivelmente, se explica pelo fato de a minoria dos profissionais de nível operacional apresentar valores de encaixe abaixo da média. Além disso, o valor da mediana para o grupo técnico-administrativo $(0,23)$ ficou abaixo do grupo operacional $(0,24)$, indicando, para a metade dos profissionais daquele grupo (técnico-administrativo), menor encaixe que a metade dos profissionais do grupo operacional.

Finalmente, o valor mais elevado de encaixe P-O encontrado para o grupo gerencial pode ser explicado pelo fato de esses profissionais serem responsáveis por operacionalizar o discurso da organização, participando da definição de suas normas e regras e zelando para que sejam praticados e seguidos pela operação. Segundo Freitas (1991), quanto mais próximos da cúpula da organização, maior tende a ser a adesão dos profissionais aos valores organizacionais.

\section{CONCLUSÕES}

Tendo por base os tipos motivacionais do modelo de Schwartz (1992), os resultados do estudo revelam que "benevolência" e "conformidade" foram considerados os dois tipos mais relevantes no contexto da organização-alvo do estudo. Já os tipos "estimulação" e "poder" apareceram nas duas últimas posições de relevância.

Em uma análise comparativa envolvendo os três grupos funcionais pesquisados registrase elevado nível de homogeneidade nas percepções de importância em relação à valores 
pessoais. Ou seja, não foram verificadas diferenças acentuadas na ordem de priorização dos tipos motivacionais: as cinco primeiras e as cinco últimas prioridades de cada grupo foram as mesmas. Tais similaridades nos perfis dos VP dos grupos funcionais investigados e, portanto, homogeneidade na população da organização, se dá a despeito dos diferentes papeis desempenhados por esses profissionais e das suas distintas características demográficas.

Quanto aos fatores de segunda ordem do modelo de Schwartz (1992), verificou-se maior distanciamento entre os grupos funcionais para a dimensão bipolar "autopromoção" versus "autotranscedência" comparativamente ao fator "autotranscedência". Tal resultado sugere que os profissionais atribuem maior importância aos valores relacionados à aceitação dos outros e à preocupação com seu bem-estar (autotranscedência) quando comparados a valores relacionados à busca do próprio sucesso e domínio sobre os outros (autopromoção).

Os resultados empíricos revelam, também, maior importância atribuída pelos profissionais de nível operacional a valores relacionados à preservação de práticas tradicionais e proteção da estabilidade (conservação), quando comparados a valores relacionados à independência do indivíduo e abertura a mudanças. Verifica-se, ainda, que o grupo gerencial se diferencia em relação aos demais grupos ao apresentar escores significativamente inferiores para os fatores "autotranscedência" e "conservação". Já os fatores "autopromoção" e "abertura a mudanças" apresentam-se, para os profissionais técnicos-administrativos, escores bastante superiores comparativamente aos demais grupos.

Quanto à mensuração dos valores organizacionais percebidos pelos três grupos pesquisados, registra-se a prevalência das categorias "domínio" e "prestígio organizacional". Já os fatores com menores escores se associam à "tradição" e ao "bem-estar do indivíduo". Portanto, os achados sinalizam um consenso entre os três grupos pesquisados quanto à prioridade atribuída pela organização a valores relacionados à busca de prestígio, admiração e respeito da sociedade, bem como ao controle e definição clara dos limites dos comportamentos de seus membros (domínio).

$\mathrm{Na}$ percepção dos três grupos pesquisados, as categorias "tradição" e "bem-estar do empregado" são as características que a organização confere menor prioridade. Tal achado indica, portanto, a baixa ênfase atribuída a valores relacionados à manutenção da cultura vigente e à qualidade de vida no trabalho, percebido, inclusive, pelos profissionais de nível gerencial.

Constatou-se ainda homogeneidade na percepção dos profissionais dos grupos operacional e técnico-administrativo, os quais apresentaram a mesma ordem de prioridade para as cinco primeiras categorias de valores organizacionais. Quanto ao grupo gerencial, as duas principais diferenças ocorreram em relação à quarta posição da categoria "domínio" e à segunda posição da categoria "preocupação com a coletividade", as quais aparecem na primeira e na quinta posição nos demais grupos, respectivamente. Tais resultados indicam, portanto, que os profissionais de grupo gerencial percebem mais ênfase da organização em valores relacionados à preocupação com o coletivo e menor ênfase em valores relacionados ao poder e ao controle sobre pessoas e recursos (domínio).

Quanto ao cálculo do encaixe $\mathrm{P}-\mathrm{O}$, contatou-se aderência entre os grupos funcionais pesquisados, evidenciando congruência entre os VP e VO acima do nível médio. Em uma análise comparativa, o encaixe $\mathrm{P}-\mathrm{O}$ apresentou-se mais elevado para os profissionais de grupo gerencial. Esse achado pode ser explicado pelo fato de os diretores/gerentes serem um dos principais agentes responsáveis por disseminarem as diretrizes, os valores e as normas organizacionais, esperando que esses elementos sejam internalizados pelas demais instâncias hierárquicas da organização. Logo, seria esperado que tais profissionais manifestassem maior congruência entre VP e VO, comparativamente aos demais.

Em suma, a partir dos resultados foi possível corroborar a viabilidade de aplicação do encaixe P-O por meio da análise da congruência entre VP e VO na organização alvo do 
estudo. Outra contribuição foi ampliar as pesquisas nacionais sob a perspectiva interacionista ou encaixe P-O, considerando a escassez de estudos sobre a congruência entre indivíduo e organização no contexto brasileiro (SANTOS, 2016). Além disso, poucas investigações analisaram o efeito combinado de construtos de natureza pessoal e situacional no desempenho organizacional (TEPECI, 2001; 2011; SANTOS, 2016), como propôs o presente estudo.

$\mathrm{O}$ estudo também amplia a literatura sobre o tema considerando potenciais implicações da transição para a quarta revolução industrial, em que novas formas de relações indivíduo-trabalho-organizações se apresentam (SUSSKIND, SUSSKIND, 2017; ROSS, 2017). Neste contexto, torna-se importante maior consideração quanto ao conteúdo dos valores (re-)produzidos na organização, bem quanto ao grau de congruência entre valores organizacionais e pessoais. Por fim, este estudo abrangeu profissionais de diferentes níveis hierárquicos da organização, superando a literatura na área que se concentrou prioritariamente nos níveis gerenciais da organização (ANDRADE; MOURÃO, 2001; CARVALHO; SOUZA, 2003).

Quanto às limitações da pesquisa, os achados empíricos representam um grupo específico de indivíduos e suas percepções sobre a organização alvo do estudo. Portanto, devem ser vistos com restrições no tocante a generalizações. Além disso, a pesquisa captou um momento estático dos indivíduos na organização, o que poderia ser possível através de um estudo de natureza longitudinal. 


\section{REFERÊNCIAS}

ALVES, Sarah Santos; CARVALHO, Virgínia Donizete de. Discutindo a adequação das escalas de mensuração de valores ao contexto das instituições sem fins lucrativos: uma análise do inventário de perfis de valores organizacionais. REAd. Rev. eletrôn. administração, Porto Alegre, v. 26, n. 3, p. 739-764, 2020.

ANDRADE, J.E.B; MOURÃO, L. Significado do Trabalho: Caminhos percorridos e sinalização de tendências. In: ENANPAD, 25, 2001. Anais... Campinas, 2001.

BAYNE, R. Psychological types at work. Cengage Learning, 2011.

BORGES, L.O. Valores de trabalhadores de baixa renda. In: TAMAYO, A.; PORTO, J.B. (Orgs.) Valores e comportamento nas organizações. 1. ed. RJ: Editora Vozes, 2005.

CARVALHO, V.; SOUZA, W.J. Qualidade de vida em organizações de trabalho voluntário: o modelo de Hachman \& Oldham aplicado à Pastoral da Criança. In: ENANPAD, 27, 2003. Anais... Atibaia, 2003.

DELFINO, I. A. L.; LAND, A. G.; RUFIN, W. A Relação entre valores pessoais e organizacionais em uma cooperativa comparados com os princípios do cooperativismo. In: XXXIV Encontro da ANPAD, 2010. Anais... Rio de janeiro, 2010.

FREITAS, M.E. Cultura organizacional: formação, tipologia e impactos. São Paulo: MacGraw-Hill, 1991.

GONÇALVES, C.A.; MEIRELLES. A. de Moraes. Projetos e relatórios de pesquisa em Administração. São Paulo: Atlas. 2004.

HAIR JR., J.F. et al. Multivariate data analysis. $5^{\text {th }}$ ed. Upper Saddle River (NJ): Prentice Hall, 1998.

KLINE, R.B. Principles and practice of structural equation modeling. New York: The Guilford Press, 1998.

KRISTOF, A.L. Person-organization fit: An integrative review of its conceptualization's measurement and implications. Personnel Psychology, v.49, p.1-49. 1996.

MARTINS, M.C.F.; SANTOS, G.E. Adaptação e validação da Escala de Satisfação no Trabalho. Psico - USF, v.11, n.2, p.195-205, 2006.

MEDEIROS, J.P.; OLIVEIRA, J.A. Uma viagem à produção científica em qualidade de vida no trabalho (QVT) nos anos 2001 a 2005: estudo nos Anais do ENAPAD. In: I Encontro de Gestão de Pessoas e Relações de Trabalho - GPR2007, Anais.... Natal-RN, p.1-14, 2007.

MEGLINO, B.M.; RAVLIN, E.C. Individual values in organizations: Concepts, controversies, and research. Journal of Management, V. 24, n.3, p.351-389, 1998. 
OLIVEIRA, A.F. Confiança do empregado na organização: impacto dos valores pessoais, organizacionais e da justiça organizacional. 2004. 259f. Tese (Doutorado) - UNB, Instituto de Psicologia, Brasília, 2004.

OLIVEIRA, A.F.; TAMAYO, A. Inventário de perfis de valores organizacionais. Revista de Administração da USP, v.39, n.2, p.129-140, 2004.

OLIVEIRA, S.; PICCININI, V.C.; FONTOURA, D.S.; SCHWEIG, C. Buscando o sentido do trabalho. In: ENANPAD, 28, 2004, Curitiba. Anais... Curitiba, ANPAD, 2004.

PASCHOAL, T.; TAMAYO, A. A relação da motivação para o trabalho com as metas do trabalhador. RAC, v.7, n.4, p.33-54, 2003.

PESTANA, M.H.; GAGEIRO, J.N. Análise de dados para ciências sociais: complementaridade do SPSS, 2 ed, Lisboa, 2003.

PRADO, K.; SANT'ANNA, A. S.; DINIZ, D. M. Múltiplos Sentidos do Trabalho no Setor de Enfermagem. In: XLIV Encontro da ANPAD, 2020. Anais... Online, 2020.

PORTO, J.B. Mensuração de valores no Brasil. In: TAMAYO, A.; PORTO, J.B. (Orgs.) Valores e comportamento nas organizações. 1. ed. RJ: Editora Vozes, 2005.

ROBERT, C.; WASTI, S. A. Organizational Individualism and Collectivism: Theoretical Development and an Empirical Test of a Measure. Journal of Management, v.28, n.4, p. 544-566, 2002. DOI: 10.1016/S0149-2063(02)00143-5.

RODRIGUEZ, C. V. O.; TOMEI, P. A.; SERRA, B. P. de C. Alinhamento pessoaorganização (P-O FIT) e engajamento: um estudo de caso. Iberoamerican Journal of Strategic Management. v. $19, \quad$ n. 1, p. 128-148, 2020. Disponível em: https://doi.org/10.5585/riae.v19i1.15899

ROHAN, M.J. A rose by any name? The values construct. Personality and Social $\begin{array}{llllll}\text { Psychology review, } & \text { v.4, n.3, } 2000 . & \text { DOI: }\end{array}$ https://doi.org/10.1207\%2FS15327957PSPR0403_4.

ROSS, A. The industries of the future. New York: Simon \& Schuster, 2017.

SANTOS, L. B.Person-organization fit: a bibliometric study and reflections. Revista Gestão \& Tecnologia, v. 16, n. 1, p. 55-81, 2016.

SCHWAB, K. A quarta revolução industrial. São Paulo: Edipro, 2016.

SUSSKIND, R.; SUSSKIND, D. The future of the professions: How technology will transform the work of human experts. Glasgow: Oxford University Press, 2017.

SANT'ANNA, A.S.; MORAES, L.F.R.; KILIMNIK, Z.M. Qualidade de vida no trabalho: o estado da arte das pesquisas no Brasil. Revista de Ciências Gerenciais, v.1, n.1, p.41-61, 2003.

SANT'ANNA, A. S.; FERREIRA, J.; SANTOS, T. C. Revolução 4.0: uma 'radiografia' de países de economia desenvolvida e do Brasil. RENI, v. 4, n. 2, p. 27-50, 2020. 
SANT'ANNA, A. S.; MORAES, L. F. R.; KILIMNIK, Z. M. Competências individuais, modernidade organizacional e satisfação no trabalho: um estudo de diagnóstico comparativo.

RAE-eletrônica, v. 4, n. 1, p. 1-23, 2005.

SCHEIN, Edgar H. Cultura organizacional e liderança. Tradução de Ailton Bonfim Brandão. São Paulo: Atlas, 2009.

SCHWARTZ, S.H. Universals in the content and structure of values: Theoretical advances and empirical tests in 20 countries. In: ZANNA, M.P. (Ed.), Advances in experimental social psychology, v.24 (pp.1-65). San Diego: Academic, 1992.

SCHWARTZ, S.H. Validade e aplicabilidade da teoria de valores. In: TAMAYO, A.; PORTO, J.B. (Orgs.) Valores e comportamento nas organizações. 1.ed. RJ: Editora Vozes, 2005.

SILVA, A.A.R. Relações entre valores organizacionais e treinamento gerencial: um estudo de caso. 2004. 137f. Dissertação (Mestrado em Administração) - UFMG, Belo Horizonte.

TAMAYO, A. et al. Prioridades axiológicas e comprometimento organizacional. Psicologia: Teoria e Pesquisa, v.17, n.1, p.27-35, 2001.

TAMAYO, A. Impacto dos valores pessoais e organizacionais sobre o comprometimento organizacional. In: TAMAYO, Á.; PORTO, J.B. (Orgs.) Valores e comportamento nas organizações. 1. ed. RJ: Editora Vozes, 2005.

TAMAYO, A.; MENDES, A.M. Valores organizacionais e prazer-sofrimento no trabalho. Psico-USF, v.6, n.1, p.39-46, 2001. DOI: https://doi.org/10.1590/S141382712001000100006.

TAMAYO, A. Prioridades axiológicas, atividade física e estresse ocupacional. RAC, Curitiba, v.5, n.3, p.127-147, 2001. DOI: https://doi.org/10.1590/S1415-65552001000300007.

TAMAYO, A.; SCHWARTZ, S.H. Estrutura motivacional dos valores humanos. Psicologia: Teoria e Pesquisa, v.9, n.2: p.329-348, 1993.

TEPECI, M.; BARTLETT, A.L.B. The Hospitality industry culture profile: a measure of individual values, organizational culture, and person-organization fit as predictors of job satisfaction, and behavioral intentions. Hospitality Management, v.21, p.151-170. 2002.

TEPECI, M. The effect of personal values, organizational culture, and personorganization fit on individual outcomes in the restaurant industry. 2001. 104f. Tese (Doutorado) - The Pensylvania State University, The Graduate School of Hotels, Pennsylvania.

TEPECI, M. The impact of person-organization fit on employee attitudes in the hospitality industry. Journal of Travel and Tourism Research, v. 11, n. 1, p. 19-35, 2011. 
VOGEL, L.; SANT'ANNA, A. S. Intuition and creativity in Brazilian executives and managers: a study based on MBTI. In: VII EnGPR. Anais... São Paulo: ANPAD, 2020. 